\title{
Hacia una sociedad del conocimiento en materia de agua en México
}

\author{
Ileana Nieves Velázquez* \\ Yolanda Alicia Villegas González**
}

Recibido: 30 diciembre 2016

\section{Resumen}

Este artículo analiza lo que la próxima Reforma a la Ley de Aguas Nacionales ya que tradicionalmente han existido conflictos de interés de las autoridades involucradas en la materia. El agua es un bien escaso y su gestión y manejo debe preocupar y ocupar a la sociedad en su conjunto. Es preciso que México se convierta en una sociedad del conocimiento en materia de agua en México y que en aras de la democracia y del cuidado a un bien procomún, desarrollemos técnicas de inclusión para la generación de ciudadanos que estén en posibilidad de obtener información relevante en torno al recurso hídrico, de filtrar tal información y de obtener más y mejor conocimiento de la misma. La propuesta que se establece en el presente artículo, es la participación de los distintos sectores poblacionales en un "Laboratorio Procomún" del agua, con el objeto de incluir las perspectivas bastas y complejas de los núcleos sociales (incluyendo la premisa de registrar como un tema trascendente en México, el implementar tecnologías innovadoras que satisfagan las necesidades de abasto y suministro a los mexicanos), y coadyuvar en la concientización sobre el cuidado del vital líquido.

Palabras clave: Escasez de agua en México, innovación, laboratorio del procomún, Reforma a la Ley de Aguas Nacionales, sociedad del conocimiento

\section{Towards a society of water knowledge in Mexico summary}

\section{Abstract}

This article analyzes what the next Mexican National Water Law Reform will participate actively in the drafting of legislative proposals in this respect since there have traditionally been conflicts of interest of the authorities involved in the matter. Water is a scarce

\footnotetext{
* Universidad Autónoma de Nuevo León. Ileana.mty@gmail.com

** Universidad Tecnológica de Monterrey. yolandavg@gmail.com
} 
commodity and its management and management must concern and occupy society as a whole. It is necessary that Mexico becomes a knowledge society on water in Mexico and that for the sake of democracy and care for a common good, we develop inclusion techniques for the generation of citizens who are in a position to obtain relevant information in The water resource, to filter such information and to obtain more and better knowledge of it. The proposal established in this article is the participation of the different population sectors in a "Common Laboratory" of water, in order to include the complex and complex perspectives of the social nuclei (including the premise of registering as a theme Transcendent in Mexico, to implement innovative technologies that satisfy the needs of supply and supply to Mexicans), and to contribute to the awareness of the care of the vital liquid.

Key words: Water scarcity in Mexico, innovation, laboratory of the commons, Reform to the Law of National Waters, knowledge society.

\section{Sumario}

I. Introducción, II. En busca de una sociedad del conocimiento en materia de agua en México, III. Promoción de una distribución equitativa del agua, IV. Diferentes mecanismos para involucrar a distintos segmentos de la sociedad para un mejor manejo y gestión del agua en México, V. Conclusión. Bibliografía.

\section{Introducción}

En la actualidad, existen importantes problemas respecto al abastecimiento del agua potable a nivel mundial y México no ha sido la excepción, "históricamente, nuestro país ha enfrentado una situación hídrica compleja, con episodios graves de sequía, desabasto o inundaciones" (Peña Nieto, 2014).El país requiere que su población cuente con agua potable, drenaje y saneamiento para garantizar su salud y para asegurar el crecimiento económico del país. La relevancia de este artículo radica en concientizar a la gente que el tema de agua hoy en día es muy importante y que no sólo se debe tener conocimiento de ello sino que la sociedad se comprometa a cuidar y a luchar por un bien necesario y agotable.

El objetivo que se presenta es demostrar que es imperante tomar conciencia de que "la adecuada disponibilidad y calidad del agua es uno de los pilares del 
desarrollo nacional, una condición necesaria para mantener el bienestar y la salud de nuestra población y uno de los elementos indispensables para un medio ambiente sano" (Abud Guerra, 2014); por ende, es trascendente invertir tiempo y esfuerzo en vislumbrar la aportación que las nuevas tecnologías pueden generar en torno a la problemática representada por la escasez del agua.

Este artículo se divide en tres partes, en la primera se tratará sobre el tema del agua en México y la importancia de la búsqueda de la aplicación de un área que mejore a las comunidades en su conjunto, exprese sus ideas y sea parte del cambio que se derive de las reformas que se implementen en materia de agua y se describirá también los beneficios de la aplicación de un "Laboratorio del Procomún"; en la segunda parte, se planteará el problema de la escasez del agua en México, y la importante y necesaria lucha por lograr una distribución equitativa del agua en el mundo. En la tercera, parte se analizarán algunos mecanismos que pueden ayudar a lograr el fomento de una sociedad del conocimiento en materia de agua y una justa distribución equitativa del agua en todo el mundo a través de la participación de los distintos segmentos de la sociedad como lo son el pueblo, los gobernantes, la industria privada y las escuelas.

Al finalizar, se concluirá con la relevancia que el agua tiene en la vida cotidiana y que es menester lograr que la sociedad civil, los órganos reguladores, los Consejos de Cuenca ${ }^{1}$, la Comisión Nacional del Agua (CONAGUA) ${ }^{2}$, los Estados, los municipios y los sectores académicos, productivos (agrícola principalmente) e industrial, entiendan a cabalidad cuáles son las mejores prácticas en torno al manejo y gestión del agua, de tal forma que apliquen en las políticas públicas y en la próxima Reforma a la Ley de Aguas Nacionales, un verdadero conocimiento y un paradigma de innovación, crecimiento y tecnología para solucionar de fondo la

1 La Ley de Aguas Nacionales establece que los Consejos de Cuenca son órganos colegiados de integración mixta, para la planeación, realización y administración de las acciones de gestión de los recursos hídricos por cuenca hidrológica o por región hidrológica. De acuerdo a la ley, constituyen instancias de apoyo, concertación, consulta y asesoría entre la Conagua y los diferentes usuarios del agua a nivel nacional. Al 31 de diciembre de 2011 había 26 Consejos de Cuenca. Para mayor información, consultar el Atlas Nacional del Agua México 2012, disponible al 4 de febrero de 2016 en la siguiente página web: http://www.conagua.gob.mx/atlas/ impacto38.html

2 La Comisión Nacional del Agua es un órgano administrativo desconcentrado de la Secretaría de Medio Ambiente y Recursos Naturales, que se encarga de la administración integral del recurso hídrico y el cuidado de su conservación. 
problemática del abasto y suministro de agua en México.

\section{En busca de una sociedad del conocimiento en materia de agua en México}

A mayor abundamiento, las circunstancias que tradicionalmente han cubierto el manejo y la gestión del agua en México, han estado mezcladas con conductas nocivas de la autoridad encargada de llevar a cabo dichas atribuciones. Es importante reconocer que existen conflictos de interés en cuanto a la infraestructura construcción de presas, acueductos, etc. y las concesiones de agua que se han otorgado en el país a lo largo de los años. De allí la importancia de que como sociedad estemos conscientes de los aspectos relevantes de la Reforma y de que pugnemos por un mejoramiento en la legislación e implementación de la misma.

En este sentido, la única forma en que podemos participar en el proceso de Reforma, es convirtiéndonos en una sociedad informada, que pueda en efecto filtrar y transformar la información que recibe en conocimiento y consecuentemente, en desarrollo. Tal y como lo describe la Organización de las Naciones Unidas para la Educación, la Ciencia y la Cultura (UNESCO) "las sociedades del conocimiento tratan principalmente de capacidades para identificar, producir, procesar, transformar, diseminar y usar la información para construir y aplicar conocimiento para el desarrollo humano. Ellas requieren de una visión social empoderante que comprenden pluralidad, inclusión, solidaridad y participación" (UNESCO, 2005: 27).

Por consiguiente, las autoridades del agua en México deben conducirse de una forma más constructiva y plural en cuanto a la participación de los distintos sectores poblacionales en la Reforma. La visión empoderante de la que trata la UNESCO al referirse a las sociedades del conocimiento, es premisa fundamental para que se disminuyan las cuotas de poder alrededor de los órganos reguladores y ejecutores de las políticas públicas del agua en nuestro país, y para que se alcance una verdadera participación ciudadana en dicha Reforma.

Con base en lo anteriormente descrito, es trascendente mencionar que la 
Constitución Política de los Estados Unidos Mexicanos ${ }^{3}$ en su artículo 4 establece textualmente lo siguiente:

“Toda persona tiene derecho al acceso, disposición y saneamiento de agua para consumo personal y doméstico en forma suficiente, salubre, aceptable y asequible. El Estado garantizará este derecho y la ley definirá las bases, apoyos y modalidades para el acceso y uso equitativo y sustentable de los recursos hídricos, estableciendo la participación de la Federación, las entidades federativas y los municipios, así como la participación de la ciudadanía para la consecución de dichos fines".

De la transcripción anterior se colige que el marco constitucional está "garantizando" el acceso al vital líquido y consecuentemente, debería incluirse dentro de las atribuciones de la CONAGUA en su carácter de órgano encargado de la gestión de los recursos hídricos, tal función. A la fecha, esta facultad no se establece en la Ley General de Aguas.

Partiendo precisamente de que la UNESCO en su Reporte denominado "Towards Knowledge Societies" del año 2005, ha manifestado que los derechos humanos son fundamentales para la existencia de una sociedad del conocimiento y que éstos deben ser la piedra angular de tales sociedades (UNESCO, 2005), desde la óptica de la propia Ley de Aguas Nacionales, ya se presenta un irrespeto a las garantías individuales de los mexicanos. Esto se genera porque la CONAGUA no tiene facultades para salir y buscar soluciones de fondo en relación con la escasez de agua en el país. Es imprescindible reflexionar la razón por la cual no hemos logrado convertirnos en una sociedad del conocimiento en materia de manejo y gestión del agua. Es claro que la actitud de los órganos reguladores ha estado marcada por la negación al uso de sistemas innovadores y de nuevas tecnologías para abatir los efectos del cambio climático y de la reducción del recurso hídrico en el país.

Paul David y Dominique Foray, en su escrito "Economic Fundamentals of the Knowledge Society", han manifestado que "los historiadores económicos han argumentado que en la actualidad, las disparidades en la productividad y en el desarrollo de los distintos países tiene menos que ver con la abundancia (o la

3 Disponible al 22 de octubre de 2016 en la página oficial de la Cámara de Diputados en la siguiente página web: http://www.diputados.gob.mx/LeyesBiblio/htm/1.htm 
falta de la misma) de recursos naturales, sino con la capacidad de mejorar la calidad del capital humano y de los factores de producción: en otras palabras, para crear conocimiento e ideas, e incorporarlas en equipos y personas" (David \& Foray, 2016).

Entonces, en México se necesita construir los puentes para mejorar el capital humano dentro de las instituciones que manejan y gestionan el agua. Para lograrlo, se puede recurrir a múltiples herramientas, entre ellas la realización de un MediaLab ${ }^{4}$ que revitalice la noción de la población en un tema "procomún", como lo es el caso del agua.

Sobre el particular, tal y como se mencionó al inicio, es importante visualizar la problemática de la escasez del agua en México como una cuestión que atañe a todos los mexicanos, y por ende, todos los miembros de la comunidad debieran tener la oportunidad de participar en lo concerniente al proceso de Reforma. Los ciudadanos pueden transformarse en una sociedad del conocimiento de los "procomunes".

Cuando se menciona que todos deben ser partícipes, debe entenderse que por lo menos los grupos más representativos de la sociedad civil (y no sólo de los grupos económicos preponderantes, como lo pueden ser las cementeras, los miembros de la $\mathrm{ANEAS}^{5}$, algunas universidades de la Ciudad de México, los grupos de agricultores y el propio Gobierno, a través de sus distintas instancias -Ejecutivo, Legislativo y Judicial-) tienen que ser escuchados a este respecto. Debe instarse a la comunidad para actuar con una moral de estructura y contenido hacia la construcción de una sociedad del conocimiento en torno a la Reforma.

Existe un área para mejorar que es relevante para que la comunidad en su conjunto exprese sus ideas y sea parte del cambio que se derive de la Reforma, para ello se propone la utilización de la fórmula del "Laboratorio del Procomún", creada por Antonio Lafuente, la cual "tiene como objetivo articular un discurso y una serie de acciones y actividades... Procomún busca expresar mediante un término nuevo una idea muy antigua: que algunos bienes pertenecen a todos y que en conjunto forman una comunidad de recursos que debe ser activamente

4 Para revisar este término y sus implicaciones, favor de remitirse a la siguiente página web, disponible al 22 de octubre de 2016: http://medialab-prado.es

5 ANEAS es el acrónimo de la Asociación Nacional de Empresas de Agua y Saneamiento de México, A.C. 
protegida y gestionada" (MediaLab Prado, 2014).

E1 "Laboratorio del Procomún" es un claro ejemplo de cómo un foro que conjunta a personas de diversos ámbitos, como lo es el del Derecho, el periodismo, la política, la ecología, el activismo, etc., coadyuva a la formación de grupos de trabajo que se reúnen periódicamente para trabajar, ya sea en línea o de manera presencial, para debatir y planificar acciones que aportan concientización en relación con el valor de los diversos "procomunes" y sobre los peligros que representan una amenaza a los mismos. La utilización de este modelo puede ser un primer paso para la construcción de una sociedad del conocimiento en materia de agua en México.

\section{Para Antonio Lafuente:}

"La noción de procomún es un concepto ancho, plural y elusivo. Ancho, porque abarca una considerable diversidad de bienes naturales (selvas, biodiversidad, fondos marinos o la Luna), culturales (ciencia, folclore, lengua, semillas, Internet), sociales (agua potable, urbe, democracia, carnaval) y corporales, también llamados de la especie (órganos, genoma, datos clínicos)".

Plural, porque son tan múltiples como los muchos modos de existencia que adoptan las comunidades, tanto en el plano local, como en el regional, estatal o internacional, pues no hay comunidad sin un procomún donde asentarse.

Elusivo, porque siendo fundamental para la vida lo tenemos por un hecho dado. Un don que sólo percibimos cuando está amenazado o en peligro de desaparición" (MediaLab Prado, 2007).

Al calce de la descripción de Lafuente, sobre el concepto de "procomún" y en conexión con el agua y con la Reforma, puede entenderse que siendo que la primera es un bien natural social con base en el cual se sustenta la vida del ser humano y que se encuentra actualmente amenazada en cuanto al uso, explotación y contaminación del recurso en México, parecería lógico aplicar este tipo de modelo ("Laboratorio del Procomún”) para examinar la Reforma y sus 
implicaciones a nivel comunitario.

¿Qué genera esta falta de visión y por ende, de implementación de ideas y tecnologías innovadoras que coadyuven al mejoramiento de las reservas de agua en México y al cuidado de las fuentes de abasto convencionales y no convencionales? Gran parte del problema es "cultural"; tal y como lo menciona Edward W. Said, la cultura “... lleva consigo no sólo venerar la propia cultura, sino también pensarla como si estuviera divorciada de... el mundo cotidiano" (Said, 1994). Es decir, la cultura política con base en la cual se está actuando para el aseguramiento y suministro de agua a la población, es errónea y es vista por las autoridades de una forma hierática.

Tal parece que la forma de pensar de los legisladores, del Presidente de la República y de la CONAGUA, es la única que puede prevalecer en materia de agua en México. Dicha noción de actuación política se encuentra totalmente divorciada de las prácticas internacionales, particularmente cuando hablamos de la generación de nuevas tecnologías para abatir los problemas de contaminación de aguas, de sobreexplotación de los mantos acuíferos y de poca rentabilidad de la infraestructura existente. ¿Dónde está la sociedad del conocimiento vinculada con dichos órganos en un bien tan "procomún" y relevante como el agua? Cabe la reflexión pues nos encontramos ante un momento crítico a nivel mundial y en específico en México.

\section{Promoción de una distribución equitativa del agua}

Es evidente que "la realidad es que la distribución del agua dulce es desigual entre las regiones naturales y económicas del planeta. Cerca del 75\% de la población humana se concentra en países y regiones donde sólo existe el 20\% de las disponibilidades de agua. Por el acelerado deterioro de los recursos acuáticos esta situación empeorará en el futuro próximo. Se espera, en efecto, que hacia el 2025, el 80\% de la población de la Tierra viva bajo condiciones de alta y muy alta escasez de recursos hídricos. Para esa época, una tercera parte de la población vivirá en situaciones consideradas como altamente catastróficas por la falta de agua" (Toledo, 2007). 
Los datos anteriores revelan la situación que estamos enfrentando a causa del mal manejo del agua que hemos realizado en los últimos 65 años; y peor aún es que dicha problemática tiende a agravarse a tal grado que, si en los próximos años no logramos extraer agua de forma sustentable de fuentes no convencionales (como las aguas subterráneas), los sectores agrícola e industrial serán objeto de serias afectaciones, al priorizarse el abastecimiento de agua para el consumo humano y otros usos domésticos. Culturas ancestrales como la maya y la azteca tenían como prioridad el cuidado del agua y basaban la fundación de sus ciudades principales en torno a fuentes convencionales de suministro de agua.

El caso actual mexicano presenta múltiples y diversas aristas. Hay que introspectar como sociedad el tema de los conflictos de interés que prevalecen en nuestras autoridades reguladoras, pero también es importante que a la par reflexionemos cómo fue que perdimos la calidad de sociedad del conocimiento en esta materia. Históricamente, los métodos propuestos por el paradigma tecnológico dominante del agua para afrontar el rápido crecimiento de las necesidades humanas, especialmente en las áreas urbanas del mundo, han sido costosos e ineficientes. Debido a ello, según la Asociación Internacional de Recursos Acuáticos, los problemas en torno del agua seguirán siendo los mayores obstáculos para el desarrollo sostenible de la sociedad humana por varias décadas más (Niemczynowicz, 2000).

Siendo que "actualmente el volumen de agua que extraemos [en la Ciudad de México] de los acuíferos es mayor que la que se recupera naturalmente por la lluvia" (Ruiz \& Ruíz), es primordial encontrar nuevas fuentes de abastecimiento de agua que permitan que los acuíferos convencionales se recuperen de forma sustentable. La forma en que podemos lograr esto es a través del uso de nuevas tecnologías que coadyuven a la extracción de aguas que se encuentran a niveles ultraprofundos. Este tipo de aguas representan un paradigma hidrológico distinto al que rige en CONAGUA, puesto que en esencia son aguas tan antiguas que han sido conformadas a raíz del agua que se inyecta en las montañas tras las lluvias; consecuentemente, no afectan al manto acuífero tradicional ni a su recarga. Se trata de aguas que finalmente descargan en el mar o que simplemente se encuentran confinadas en mega acuíferos subterráneos ultraprofundos.

A mayor abundamiento, Ismael Aguilar Benítez expresa que 
"inevitablemente, el uso del agua perjudica la disponibilidad y la calidad de este recurso natural. En efecto, tanto una extracción no sostenible de agua de las fuentes naturales (ríos, lagunas, lagos, manantiales, acuiferos, etcétera) como la contaminación que se genera y se deposita, muy frecuentemente, en esas mismas fuentes las deterioran" (Aguilar Benítez, 2011). Por su parte, la Investigadora del Colegio de México Judith Domínguez Serrano, se ha pronunciado en el sentido de que "el reconocimiento constitucional del derecho humano de acceso al agua exige cambios en la actual gestión de los servicios públicos del país, para superar los problemas técnicos y políticos en los que se encuentran inmersos cada trienio municipal la mayoría de los organismos operadores" (Domínguez Serrano, 2014).

\section{Diferentes mecanismos para involucrar a distintos segmentos de la sociedad para un mejor manejo y gestión del agua en México}

Con base en lo anterior, es innegable la necesidad de construir una visión plural y efectiva para el manejo y la gestión del agua en México. Para ello puede iniciarse con la implementación de un "Laboratorio del Procomún", de tal forma que se involucre a distintos segmentos de la sociedad en torno a la Reforma y se empiece a procesar y filtrar la información adecuada, para sobre ella crear conocimiento y sembrar las bases de la legislación del agua.

Es importante que se usen las tecnologías de la información de la sociedad actual para desarrollar una nueva forma de pensamiento y de educación. La gestión del conocimiento se vuelve clave para el desarrollo económico sustentable, se requiere generar condiciones donde el conocimiento del agua sea como una entidad cultural en relación con la distribución del mismo en México tal y como lo establece Joel Mokyr al afirmar que "lo que hace que el conocimiento sea una entidad cultural, entonces, es el que sea distribuido a, compartido con, y adquirido de otros; si dicha adquisición se convierte en algo muy difícil de hacer, el conocimiento no estará accesible a aquellos que no lo tienen pero que quieren aplicarlo" (Mokyr, 2016). En un tema "procomún" como lo es el agua y dadas 
las condiciones ambientales generalizadas en México, es imperativo hacer del conocimiento público la situación y el grave fenómeno que se avecina si no se actúa de inmediato.

Asimismo, debe entenderse que "el conocimiento es necesario para generar cambio y que el nuevo conocimiento es agregado a la fábrica social precisamente a partir de este cambio. El cúmulo de recursos y de potencialidades humanas y socio-culturales en conjunto con estrategias, políticas, creatividad e innovación, imaginación y visión, convierte a las sociedades en líderes" (Zacher, 2016). Por eso, es preciso enfocarnos en esta Reforma para fomentar la inclusión social en las políticas públicas y en la implementación de las mismas. Además, sería relevante dejar a un lado sesgos culturales y relativismos sobre el uso de tecnologías innovadoras en el campo del agua en México. Al plantear esto, es menester asegurarnos del buen uso de los capitales intangibles con los que cuenta la CONAGUA y demás entes reguladores y por qué no, con los que cuenta la sociedad civil. La ponderación de valores tiene que ser visualizada en sentido retrospectivo y prospectivo. El país no puede darse el lujo de continuar construyendo grandes infraestructuras que no son funcionales a largo plazo y que no solventan los grandes problemas de escasez de agua. Hay que crear sociedades sabias e inteligentes en el manejo y la gestión del recurso hídrico bajo principios básicos en los que todos podemos colaborar.

Debe dejarse a un lado las sociedades del entretenimiento, donde los distintos sectores de la población se dejan llevar por la información que otorgan las autoridades y donde existe distorsión de los hechos reales, de los niveles de escasez de agua, de los conflictos de interés con las cementeras y demás contratistas que construyen la infraestructura que dicta la CONAGUA. Hay que recordar que "el auge de lo divertido y la posmodernidad... llegó acompañada de un descenso de nivel" (Martínez, 2011). Es importante que la sociedad civil esté enterada de lo que ocurre en materia de agua en México y que actúe para proteger a ese recurso natural sin el cual no se puede vivir. Así de importante es que se regrese a los orígenes milenarios del cuidado del agua.

En relación con las sociedades del entretenimiento que queremos evitar, María de Lourdes López Aguirre ha comentado que "la industria del entretenimiento encaja más en la lógica de la cultura masificada que en la alta 
cultura: son poderosos mecanismos que proveen a grandes públicos de espacios para satisfacer una necesidad lúdica, recreativa y de evasión" (López, 2014) y a partir de ello puede efectuarse una analogía con respecto a la cultura masificada en torno al manejo y la gestión del agua, representada por la supuesta imperiosa necesidad de construir más y mejor infraestructura, versus la alta cultura de los innovadores que ofertan nuevas tecnologías para satisfacer las necesidades de agua del país; mientras tanto, los ciudadanos en general optan por permanecer en una sociedad del entretenimiento, recreándose con mecanismos lúdicos y de evasión hacia los problemas que en el presente y en el futuro cercano vendrán por la escasez del agua. Esto debe cambiar, tenemos que transformarnos en una sociedad del conocimiento, informada y con capacidad de filtrar tal información para entonces poder exigir derechos y pugnar por el bien social.

Actualmente existe la preocupación por incentivar la utilización de nuevas tecnologías para hacer más eficiente el uso y el suministro de agua, y tal y como lo señala el colombiano Roberto Restrepo, Coordinador del Programa Cultura y Agua para América Latina de la Organización de las Naciones Unidas para la Educación, la Ciencia y la Cultura (UNESCO), "la humanidad enfrenta ya el problema del estrés hídrico que adquirirá una dimensión crítica en el año 2025" (Boletínes UAM, 2015). Si los problemas para garantizar el suministro del agua son un riesgo inminente, tenemos que emanciparnos de los mitos tradicionales de la modernidad en materia de agua, los cuales, como lo menciona Walter D. Mignolo, han sido construidos a través de macronarrativas desde una perspectiva colonial, lo que se traduce en el hecho de que dichas macronarrativas resulten falsas porque no contenían lo que los discursos colonialistas no querían abordar (Mignolo, 2000). Esto se refleja en la toma de decisiones de inversión en infraestructura y arroja múltiples interrogantes en torno a si dichas determinaciones son realizadas por conflictos de intereses que las grandes cementeras del país generan.

En esta línea de pensamiento, la próxima Reforma representa un hito en la historia normativa de México y una vital oportunidad para cambiar el manejo que se ha dado en los últimos veinte años a la actividad de la CONAGUA y de los órganos reguladores referente a garantizar el suministro del recurso hídrico a la población. Tal y como lo ha mencionado Manuel Herrera Viga, Presidente de la Confederación de Cámaras Industriales de los Estados Unidos Mexicanos 
(CONCAMIN), el sector industrial mexicano cuenta con propuestas y necesidades que deben ser consideradas en la modernización del marco legal federal en materia de agua, al ser la industria el sector usuario con mayor responsabilidad asumida en el pago de los derechos por el uso, explotación y aprovechamiento de este recurso (Herrera, 2015). Dichas propuestas deben ser analizadas a la luz de encontrar soluciones de fondo a los problemas de escasez de agua de nuestro país, de tal forma que contemos con un sistema de aguas nacionales que responda a un proyecto de país de largo plazo.

Respecto a lo anterior, Eduardo Viesca, Presidente de la Comisión de Agua y Coordinador de Ecosistemas de Servicios de la CONCAMIN, promueve la importancia de contar con (I) una política pública en materia de agua, (II) un nuevo sistema financiero que propicie un uso eficiente, incentivándolo y sancionando el desperdicio ostensible, (III) una revisión a las vedas, zonas reglamentarias, acuiferos y construcción de plantas de tratamiento, (IV) una nueva y moderna norma oficial mexicana en materia de disponibilidad de agua y (V) un programa de apoyo a los usuarios agrícolas para detonar el uso eficiente y sustentable en el campo mexicano. (Viesca, 2015) Pero para convertirnos en una verdadera sociedad del conocimiento en materia de agua, es necesario plantearnos la siguiente pregunta ¿Cuánta agua nos queda? Actualmente se habla de una escasez que afecta a toda la tierra y de soluciones que perjudican a millones de personas, entonces, ¿qué hacemos para enfrentarla? En 2010, la Asamblea General de la ONU a través de su resolución 64/292 reconoció el derecho humano al agua y al saneamiento (Gutiérrez Bracho, 2015). En este sentido, el artículo 4 constitucional consagra tal derecho fundamental, e incluso la Suprema Corte de Justicia de la Nación ha reconocido el derecho "pro persona", lo que significa que debe en todo momento, tratándose de un tema relacionado a un derecho humano o fundamental, atenderse a la interpretación más favorecedora para el ser humano, ya sea que provenga del ámbito internacional o nacional; esta aplicabilidad sería ajustable al derecho al agua para los mexicanos. No obstante, mientras no existan los mecanismos adecuados a nivel cultural y gubernamental para el cuidado del agua, será muy complicado solventar la escasez hídrica.

Para entender la trascendencia del buen filtrado de información provista al ciudadano, cabe mencionar que en el Atlas del Agua en México 2015, la 
CONAGUA realiza aseveraciones en cuanto a que se debe tener especial cuidado con la explotación del agua subterránea, puesto que su sobreexplotación puede ocasionar afectaciones difícilmente reversibles para el ecosistema y la sociedad y a su vez, resalta el hecho de que la población rural depende de manera significativa del agua subterránea y en algunas zonas áridas la dependencia es total. (Gobierno de la República, SEMARNAT, CONAGUA, 2015)

A raíz de estos enunciados de la CONAGUA, es preciso clarificar que la regulación del agua en México no ha logrado identificar otro segmento o porción de subsuelo que no implica aguas subterráneas sino que contiene megacuencas que no presentan impacto en los mantos acuíferos tradicionales, y que tampoco afectan las aguas subterráneas; y es en ese nicho (fuente no convencional) en donde nuevas tecnologías para la exploración y extracción de aguas ultraprofundas, facilitan el acceso al recurso hídrico. Esto sin afectar a las fuentes convencionales de agua en México. Mediante la Reforma, se pueden incorporar elementos jurídicos que promuevan el uso de dichas nuevas tecnologías para solucionar los problemas de escasez del recurso hídrico.

Hay que aprender de los errores del pasado y de incentivar la búsqueda de soluciones de fondo para el cabal abastecimiento de agua en México. Entonces, ¿cómo lograr tal solución en la post-modernidad mexicana? Seyla Benhabib ha expresado que: "entre los legados de la modernidad que al dia de hoy requieren reconstruirse pero no desarmarse del todo, se encuentran la moral y el universalismo político, comprometidos con lo hoy visto 'pasado de moda' y con los sospechados ideales de respeto universal para cada persona en virtud de su humanidad; la autonomía moral del individuo; la justicia económica y social y la equidad; la participación democrática; las libertades civiles y políticas más amplias que sean compatibles con los principios de justicia; y la formación de asociaciones humanas solidarias" (Benhabib, 1992). En este tenor, hay partir de los legados antes señalados para reflexionarlos, analizarlos y mejorarlos en aras del bienestar social. De forma analógica a lo esgrimido por Benhabib, podría argumentarse que, los legados de la gestión del agua en México responden a circunstancias antiguas que ya no coinciden con la realidad ni con las necesidades actuales y futuras en los sectores agrícola, energético, industrial y poblacional; consecuentemente, deben retomarse los valores positivos 
desarrollados en la modernidad, como lo son la justicia, el respecto al individuo y a las garantías individuales (en ellas se incluye el derecho al abastecimiento de agua) y la formación de asociaciones civiles en pro de la protección al recurso hídrico, redefiniéndolos conforme a las condiciones imperantes en la actualidad y progresándolos hacia un nuevo paradigma donde se logre implementar las mejores prácticas hídricas a nivel mundial que incluyen sin lugar a dudas, el uso de innovaciones tecnológicas para la explotación del vital líquido.

\section{Conclusión}

Con base en lo expuesto y dado que "mientras que la disponibilidad anual de agua y la precipitación de un año para otro es todavía desconocida, sólo hay una cosa que es segura: que el crecimiento de la población es inevitable" (Johnson J., 2014), es fundamental el estudio de las circunstancias y del fenómeno antes descritos para que la población sea capaz de dilucidar y proponer nuevas alternativas de índole normativa y tecnológica, que permitan atacar eficazmente el problema de la escasez del agua en México. Tal y como lo afirma Michael Klare en su libro Guerras por los Recursos, "el agua presenta mucha semejanza con el petróleo. Es indispensable para una gama muy amplia de actividades humanas, y existe en cantidad limitada. Una vez agotadas las posibilidades actuales, procurarse cantidades adicionales va a requerir esfuerzos heroicos y costosos. Para ambos recursos, el aumento demográfico y la creciente prosperidad disparan la demanda mundial y cada día que pasa nos hallamos más cerca del nivel de escasez a escala planetaria. El agua es absolutamente necesaria para la salud y la supervivencia humana: para beber, para la higiene y la salubridad y para la producción de alimentos. Según el Banco Mundial, la cantidad mínima diaria que necesita un ser humano para vivir en buen estado de salud es de 100 a 200 litros. En el 2020 se esperará una población total mundial de unos 8,000 millones de habitantes. La cuestión es: ¿nuestro planeta podrá suministrar tanta agua?" (Klare, 2003).

La respuesta a la interrogante de Klare es en sentido negativo. $\mathrm{Ni}$ el mundo ni México podrán afrontar tales retos si no efectúan acciones desde hoy que permitan garantizar de forma real y sustentable el suministro de agua en 
las cantidades y calidad necesarias que requieren la población y el desarrollo económico de nuestro país.

Finalmente, instamos a que encontremos como sociedad mecanismos de acción para involucrar a los ciudadanos en la Reforma, ya sea a través de esquemas como el "Laboratorio del Procomún" o en virtud de cualquier otra herramienta a nuestro alcance que nos potencialice para convertirnos en una verdadera sociedad del conocimiento en materia de agua y en donde el humanismo sea fundamental para transformar la gestión del vital líquido en México. Los ciudadanos tienen el deber cívico de pasar de una simple moral como estructura a una moral de contenido, que les lleve a la consecución de metas que engrandezcan y amparen los recursos naturales de México, especialmente tratándose del agua, pues ésta representa un bien insustituible y necesario para la perpetuidad de la especie humana y del medio ambiente.

\section{Bibliografía}

Abud Guerra, J. J. (Abril de 2014). Mensaje. Programa Nacional Hídrico 2014-2018. Plan Nacional de Desarrollo 2013-2018. México, D.F., México: Gobierno de la República.

Aguilar Benítez, I. (2011). Los servicios del agua en el norte de México: Gestión, manejo financiero y aspectos ambientales. México, D.F.: El Colegio de la Frontera Norte; El Colegio de Sonora.

Benhabib, S. (1992). Situating the Self: Gender, Community and Postmodernism in Contemporary Ethics. New York: Routledge.

Boletines UAM. (07 de Mayo de 2015). Boletines UAM. Recuperado el 23 de Enero de 2016, de Estrés Hídrico con Dimensión Crítica para el Año 2025: http://www.comunicacionsocial.uam.mx/ boletinesuam/155-15.html

David, P.A., \& Foray, D. (22 de Octubre de 2016). Economis Fundamentals of the Knowledge Society. Stanford Economics Department Faculty Papers Archive, 3. Obtenido de Stanford Economics Department Faculty Papers Archive: http:/www-siepr.stanford.edu/workp/ 
swp02003.pdf

Gobierno de la República, SEMARNAT, CONAGUA. (2015). Atlas del Agua en México 2015. México, D.F.: SEMARNAT y CONAGUA. Gutiérrez Bracho, C. (Noviembre-Diciembre de 2015). Agua: ¿Cuánta nos queda? Tec Review, pág. 33.

Herrera, M. (11 de Septiembre de 2015). Página Web de la CONAGUA. Recuperado el 04 de febrero de 2016, de Conagua y CONCAMIN unen esfuerzos para un mejor aprovechamiento de las aguas nacionales: http://www.conagua.gob.mx/SalaPrensa. aspx $? \mathrm{n} 1=21363 \& \mathrm{n} 2=\mathrm{NotaP}$

López, M. (7 de Julio de 2014). La sociedad del entretenimiento, ¿una fábrica de incultos? Obtenido de Istmo Liderazgo con Valores: http:// istmo.mx/index.php/2014/07/07/la-sociedad-del-entretenimientofabrica-de-incultos/

Martínez, J. (2011). Sociedad del Entretenimiento (2): Construcción socio-histórica, definición y caracterización de las industrias que pertenecen a este sector. Revista Luciérnaga. Facultad de Comunicación Audiovisual. Politécnico Colombiano Jaime Isaza Cadavid, págs. 2-11.

MediaLab Prado. (30 de Agosto de 2007). Qué es el Procomún. Recuperado el 25 de Abril de 2016, de MediaLab Prado: http:// medialab-prado.es/article/video_que_es_el_procomun

MediaLab Prado. (2014). Laboratorio del procomún. Recuperado el 25 de Abril de 2016, de MediaLab Prado: http://medialab-prado.es/ laboratorio_del_procomun

Mignolo, W. D. (2000). Local Histories/Global Designs: Coloniality, Subaltern Knowledges and Border Thinking. New Jersey: Princeton University Press.

Mokyr, J. (2016). The Gifts of Athena Historical Origins of the Knowledge Economy. Princeton, New Jersey: Princeton University Press.

Niemczynowicz, J. (2000). Present Challenges in Water Management. A Need to See Connections and Interactions. Water International, págs. 139-147. 
Ruiz, R., \& Ruíz, G. (s.f.). Sobreexplotación y Análisis Multitemporal del Acuífero de la Ciudad de México. Recuperado el 02 de Abril de 2016, de Red del Agua UNAM: http://www.agua.unam.mx/ assets/3eua/pdf/carteles/cartel_rosioruiz.pdf

Said, E. W. (1994). Culture and Imperialism. New York: Vintage Books. Toledo, A. (27 de Agosto de 2007). Instituto Nacional de Ecología de la SEMARNAT. Recuperado el 23 de Enero de 2016, de El Agua en México y el Mundo: http://www2.inecc.gob.mx/publicaciones/ gacetas/366/toledo.html

UNESCO. (2005). Towards Knowledge Societies. Condé-sur-Noiré, France: UNESCO Publishing.

Viesca, E. (11 de Septiembre de 2015). Página Oficial de la CONAGUA. Recuperado el 04 de febrero de 2016, de Conagua y CONCAMIN unen esfuerzos para un mejor aprovechamiento de las aguas nacionales: http://www.conagua.gob.mx/SalaPrensa. aspx?n1 $=21363 \& \mathrm{n} 2=$ NotaP

Zacher, L. W. (23 de Octubre de 2016). Modeling Knowledge Society. Obtenido de Information Resources Management Association: http:// www.irma-international.org/viewtitle/112417/ 